\title{
Association between gut microbiota, bone metabolism, and fracture risk in postmenopausal Japanese women
}

\author{
D. Ozaki ${ }^{1}$ (D) $\cdot$ R. Kubota ${ }^{1}$ (D) T. Maeno $^{2}$ (D) $\cdot$ M. Abdelhakim $^{3}$ (D) $\cdot$ N. Hitosugi ${ }^{4}$ (D)
}

Received: 26 May 2020 / Accepted: 2 November 2020 / Published online: 25 November 2020

(C) The Author(s) 2020

\begin{abstract}
Introduction We investigated the relationship between gut microbiota composition and osteoporosis/fracture risk in Japanese postmenopausal women using 16S rRNA gene sequencing, FRAX, bone mineral density, biochemical bone parameters, and a self-administered questionnaire. Variation in abundance of specific microbiota was found to be significantly associated with fracture risk and vitamin $\mathrm{K}$ levels.

Gut microbiota data with respect to bone metabolism and fracture risk is limited. Vitamin $\mathrm{K}$ is produced by certain intestinal bacteria and has been reported to play a role in maintaining bone quality.

Purpose We investigated relationships among gut microbiota composition, bone metabolism, and fracture risk in postmenopausal Japanese women.

Methods Bone mineral density (BMD) was evaluated in 38 postmenopausal women (mean age 62.9 years) using forearm dualenergy X-ray absorptiometry. We collected and analyzed serum bone turnover markers (vitamin K fraction and tartrate-resistant acid phosphatase 5b; TRACP-5b), gut microbiota profiling (16S rRNA gene sequencing), and self-administered questionnaire data, including fracture history and vitamin $\mathrm{K}$ intake. Vitamin K2, BMD, and TRACP-5b data were divided into high- and lowlevel groups using cutoff values of $0.06 \mathrm{ng} / \mathrm{mL}, 87.05 \%$, and $420 \mathrm{mU} / \mathrm{dL}$, respectively; the proportions of bacteria were analyzed. Fracture incidence and relative risk were investigated for each bacterium.

Results The genus Bacteroides was predominant in the high vitamin $\mathrm{K} 2$ group $(29.73 \%$ vs $21.58 \%, P=0.022)$. Fracture incidence was significantly higher in the low Bacteroides group, with a 5.6-times higher risk ratio of fracture history.

The family Rikenellaceae was more abundant in the low BMD group and more abundant in the high TRACP-5b group (2.15\% vs $0.82 \%, P=0.004 ; 2.38 \%$ vs $1.12 \%, P=0.013$, respectively).

Conclusion Bacteroides and Rikenellaceae may be involved in bone metabolism and fracture risk. Further investigations of the underlying microbiota-related pathways in bone metabolism may reveal treatment strategies, and facilitate the prevention of osteoporosis.
\end{abstract}

Keywords Bone metabolism $\cdot$ Fracture $\cdot$ Gut microbiota $\cdot$ Osteoporosis $\cdot$ Vitamin $\mathrm{K}$

D. Ozaki

daiya@nms.ac.jp

1 Musashiurawa Orthopedics and Internal Medicine Clinic, Musashiurawa Medical Center, Saitama, Japan

2 Musashi Pharmacy, Musashiurawa Medical Center, Saitama, Japan

3 Department of Plastic, Reconstructive, and Aesthetic Surgery, Nippon Medical School, Tokyo, Japan

4 Muse Dermatology \& Pain Clinic, Musashiurawa Medical Center, Saitama, Japan

\section{Introduction}

In recent years, the Japanese population has been rapidly aging with the elderly population aged 65 years and above accounting for more than $27.7 \%$ of the total population. Thus, the incidence of osteoporosis is steadily increasing. The prevalence of osteoporosis in Japanese patients older than 40 years was reported to be $3.4 \%$ in men and $19.2 \%$ in women, in addition to $12.4 \%$ and $26.5 \%$ in men and women with an osteoporotic lumbar vertebral L2-L4 region, respectively [1]. The estimated number of patients with osteoporosis nationwide has exceeded 13 million, particularly in women. In postmenopausal women, osteoporosis progresses due to 
increased bone resorption due to estrogen deficiency and decreased bone density. Osteoporosis is widely considered a major risk factor for fractures. Osteoporosis-related fractures, such as fractures of the vertebral body, forearm, and proximal femur, are thus more likely to occur [2]. The Fracture Risk Assessment Tool (FRAX ${ }^{\circledR}$ ) has been developed by the World Health Organization to assess the 10-year probability of major osteoporotic fracture in patients at risk of hip fractures and major osteoporotic fractures by evaluating bone mineral density (BMD) and other risk factors relating to BMD and lifestyle [3]. The Japanese practice guidelines on osteoporosis consider fracture risk $\geq 15 \%$ as the criterion for initiating pharmaceutical treatment. Hip fracture increases mortality risk by 10 to $20 \%$ within 1 year after the fracture. Functional status of patients with hip fracture progressively worsens in $60 \%$ of these patients compared with functional status before the fracture. In addition, vertebral fractures contribute to increased risk of mortality even among those detected using radiography. Among the Japanese osteoporotic population, hip fracture occurs in 130,000 of these patients annually; of these, 20,000 patients die and 60,000 experience functional decline [4]; thus, prevention has become an important issue not only in terms of medical treatment but also for society at large.

The human microbiome consists of an estimated 100 trillion microbes, and the intestines are host to approximately 1000 different bacterial species $[5,6]$. This intestinal bacterial population is collectively referred to as the gut microbiota.

The human gut microbiota comprises 4 major co-existing phyla, including Firmicutes, Bacteroidetes, Actinobacteria, and Proteobacteria. These phyla represent over $90 \%$ of the gut microbiota [7].

The gut microbiota maintains intestinal homeostasis via a complex mechanism. Even if this homeostasis is somewhat disturbed by various stimuli, such as stress, aging, or other external factors, it has a strong tendency to return to the original condition. Moreover, oral ingestion of live beneficial bacteria (probiotics) has the potential to alter the gut microbiota. However, disruption of intestinal homeostasis (referred to as microbial dysbiosis), due to either host genetic predisposition or excessive influence of external environmental factors, can lead to loss of innate intestinal tract defense against infection. This can ultimately result in gastrointestinal disorders, such as inflammatory bowel disease (IBD) [8] and colorectal cancer [9], metabolic diseases, such as obesity [10] and diabetes mellitus [11], and various other disorders, such as depression [12], Parkinson's disease [13], and allergies [14]. This association between microbial dysbiosis and the above-mentioned diseases has been widely reported.

However, the effect of gut microbiota alteration in patients with osteoporosis remains unknown. Wang et al. analyzed the diversity of gut microbiota in patients with primary osteoporosis, osteopenia, and in normal controls. They found that in patients with osteoporosis, the proportion of Firmicutes was increased compared with that of the normal control group, and the proportion of Bacteroidetes was reported to be significantly decreased in patients with osteoporosis [15].

In terms of classification, intestinal bacteria are subdivided into class, order, family, and genus from phyla represented by Firmicutes, Bacteroidetes, Actinobacteria, and Proteobacteria (Fig. 1).

Even in the same phylum, each group such as family or genus has various functions.

For example, the Firmicutes family Ruminococcaceae consists of some butyric acid-producing bacteria such as Faecalibacterium and Butyricicoccus. Butyrate-producing bacteria are promising probiotic candidates targeting microbiota modulation in gastrointestinal disorders such as IBD [16]. Similarly, Ruminococcus gnavus, a member of the Ruminococcaceae family, has been reported to be implicated in infection following artificial hip joint replacement [17], liver abscess due to Ruminococcus gnavus, and infective endocarditis. In addition, reports have shown that Ruminococcus is involved in the development of cerebral and myocardial infarction [18]. Thus, bacteria with different functions constitute the same population in terms of classification.

This study was conducted to clarify the effects of changes in the gut microbiota, especially at the family and genus levels, in patients with osteoporosis. A better understanding of the roles of gut microbiota may lead to the development of new therapies for osteoporosis. Thus, we aimed to determine whether particular gut microbes are associated with osteoporosis by investigating and analyzing gut microbiota and bone metabolism in postmenopausal Japanese women.

\section{Methods}

\section{Subjects}

In this study, data were collected from 38 postmenopausal women (mean age 62.9 (range, 50-82) years) who visited the Musashiurawa Orthopedic and Internal Medicine Clinic for outpatient treatment from January 2017 to April 2017. All subjects provided informed consent to participate, and sufficient ethical consideration was given to avoid personal identification of each participant's data. Those with diabetes, malignant tumors, fracture in the acute phase (within 2 months), and IBD, or those who had undergone dialysis or had taken medication for osteoporosis, as well as warfarin, steroids, and antibiotics within 3 months before sample collection, were excluded. This was because these conditions and drugs could possibly affect bone metabolism and gut microbiota.

BMD was measured on the proximal $1 / 3$ of the forearm using dual-energy X-ray absorptiometry (DCS 600EXV; Hitachi Aloka Medical, Tokyo, Japan). Bone density was 


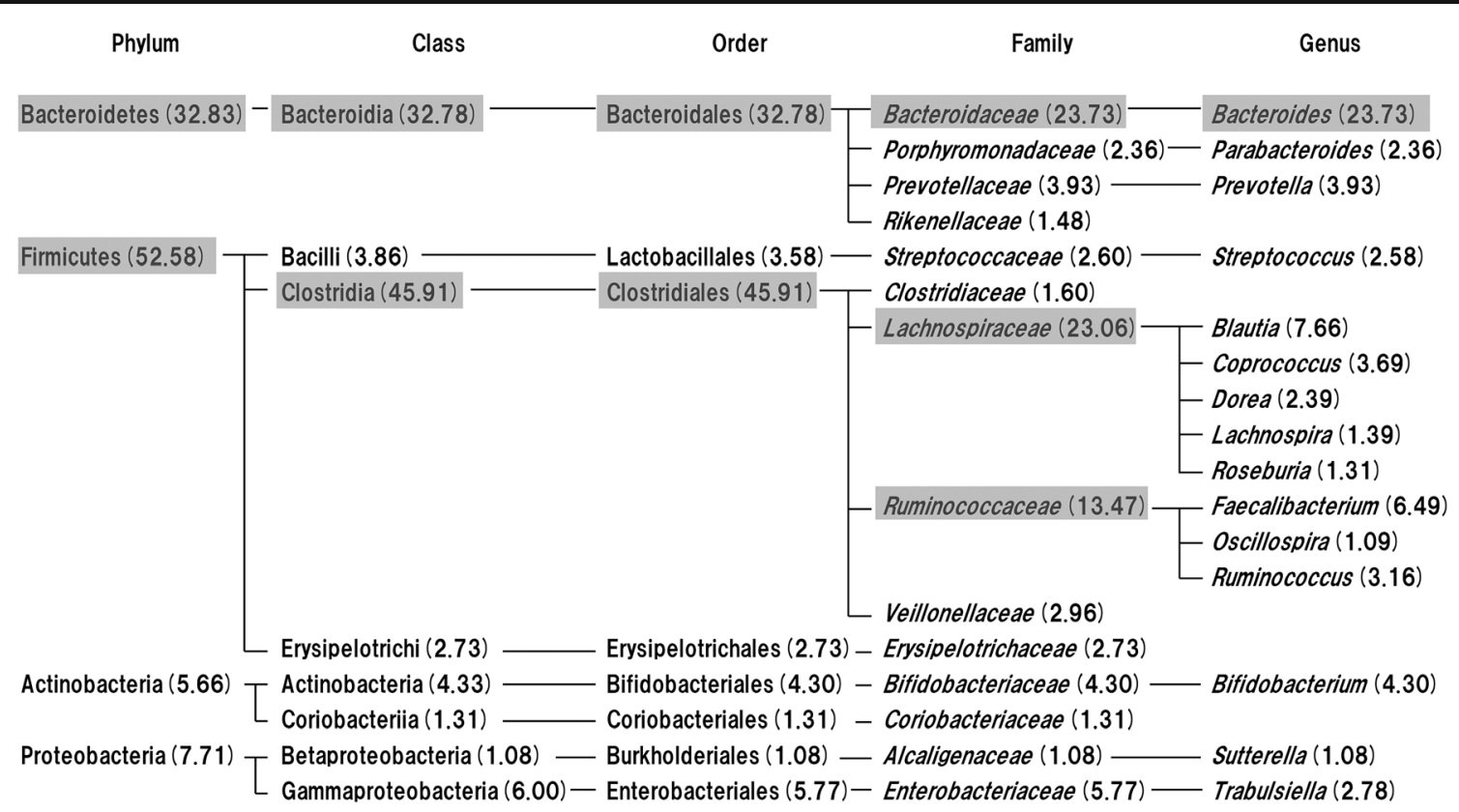

Fig. 1 Bacterial species targeted for statistical analysis. List of bacteria with their taxonomical classification (phylum-class-order-family-genus) with a mean value of $>1 \%(n=38)$. Numbers are presented as averages

determined based on the Young Adult Mean (YAM: 100\% of mean value bone density at age $20-44$ years) value.

\section{Biochemical parameters}

Due to the setup of the clinic, it was difficult to unify the timing of blood sampling, and so we opted to use tartrateresistant acid phosphatase $5 \mathrm{~b}$ (TRACP-5b) as the bone resorption marker, because it has less diurnal variation than the conventional carboxy-terminal collagen crosslinks [19]. Also, we used undercarboxylated osteocalcin (ucOC) as a measure of vitamin $\mathrm{K}$ deficiency; serum levels of vitamins $\mathrm{K} 1, \mathrm{~K} 2,1 \alpha$, 25- $(\mathrm{OH}) 2$ vitamin $\mathrm{D}$, alkaline phosphatase (ALP), calcium $(\mathrm{Ca})$, and phosphorous $(\mathrm{P})$ were measured as biochemical indices of bone metabolism.

\section{Questionnaire survey}

Menopausal age, fracture history, and FRAX score were recorded. To clarify the relationships with bacterial composition, we conducted a randomized questionnaire survey on exercise frequency, defecation frequency, frequency of alcohol intake, and dietary intake of grains, vegetables, yogurt, lactic acid bacteria beverages, and a fermented soybean product (natto), a rich source of vitamin $\mathrm{K} 2$ that is common in Japan. No dietary restrictions or lifestyle control measures were applied to the subjects during the study. Two groups were defined: a high intake group and a low intake group. Each group was surveyed about the number of meals per week $(0,1-3,4-6$, daily) and the frequency of natto intake.
$(\%)$. Bacterial species highlighted in gray indicate bacterial species with mean value of $\geq 10 \%$

Regarding fracture history, high-energy injuries such as those resulting from traffic accidents and falls from a height were excluded. Relationships between the questionnaire information and gut microbiota were analyzed statistically (Table 1).

\section{Analysis of the gut microbiota: fecal sampling, DNA extraction, and sequencing}

Fecal samples were collected using a brush-type collection kit containing guanidine thiocyanate solution (Feces Collection kit ${ }^{\circ}$ Techno Suruga Laboratory, Shizuoka, Japan) and stored at $4{ }^{\circ} \mathrm{C}$ until analysis. DNA was extracted from fecal samples using an automated DNA extraction machine (GENE PREP STAR PI-480; Kurabo Industries Ltd., Osaka, Japan) according to the manufacturer's instructions. The $16 \mathrm{~S}$ ribosomal RNA (rRNA) regions (V1-V2) were amplified using a forward primer (16S_27Fmod: TCG TCG GCA GCG TCA GAT GTG TAT AAG AGA CAG AGR GTT TGA TYM TGG CTC AG) and reverse primer (16S_338R: GTC TCG TGG GCT CGG AGA TGT GTA TAA GAG ACA GTG CTG CCT CCC GTA GGA GT) with KAPA HiFi HotStart ReadyMix PCR kit (Roche, Basel, Switzerland). To sequence 16S amplicons using the Illumina MiSeq platform (Illumina, San Diego, CA), dual index adapters were attached using the Nextera XT Index kit (Illumina). Each library was diluted to $5 \mathrm{ng} / \mu \mathrm{L}$, and equal volume aliquots were mixed to generate a library pool of $4 \mathrm{nM}$ each. The DNA concentration of the mixed libraries was quantified using qPCR with the KAPA SYBR FAST qPCR Master mix (KK4601, KAPA Biosystems, Wilmington, MA) using primer 1 (AAT GAT 


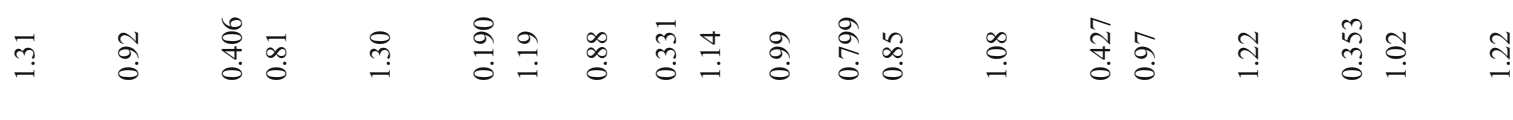

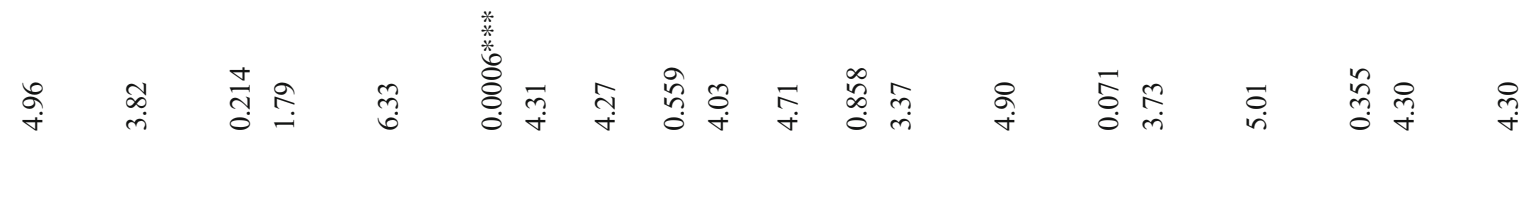

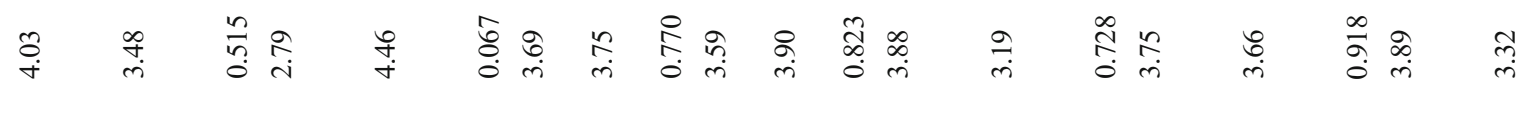

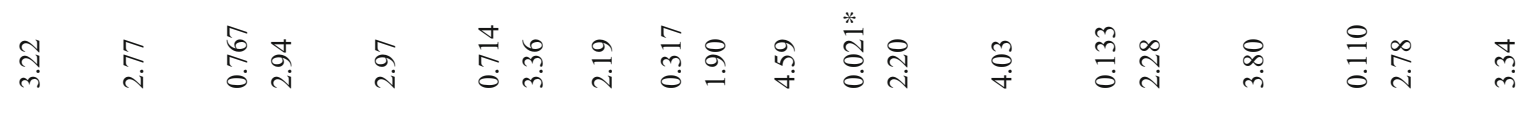

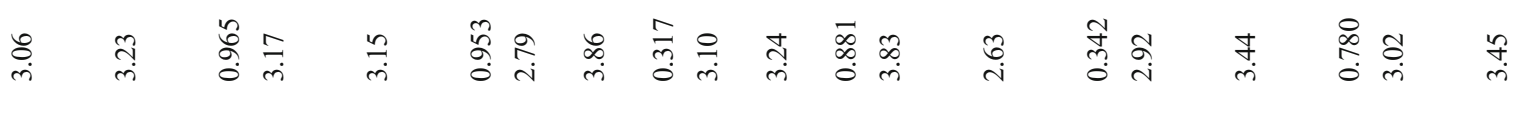

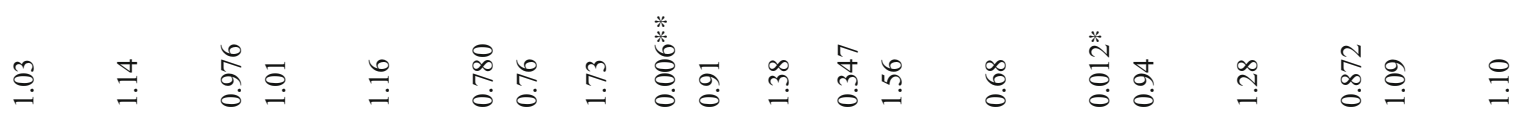

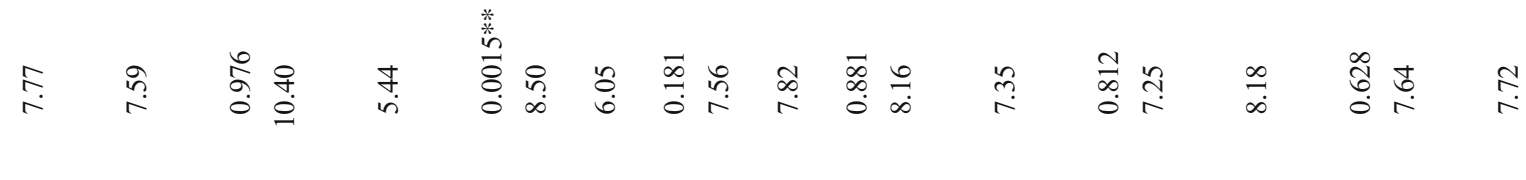

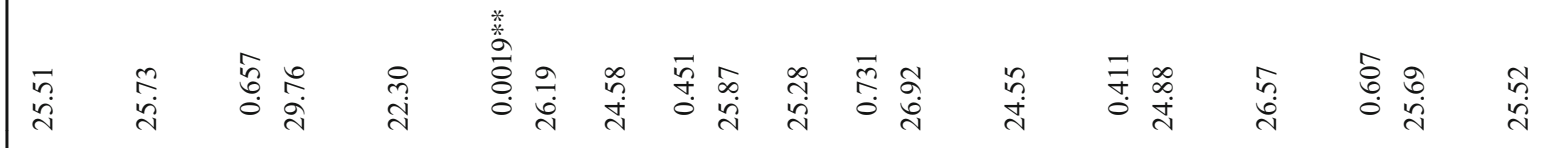

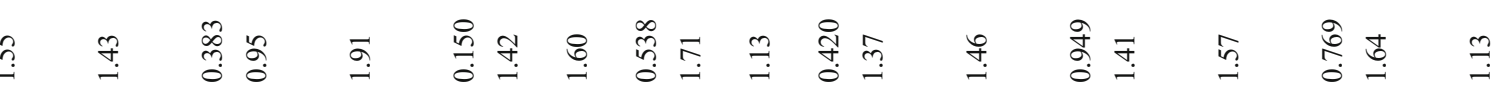

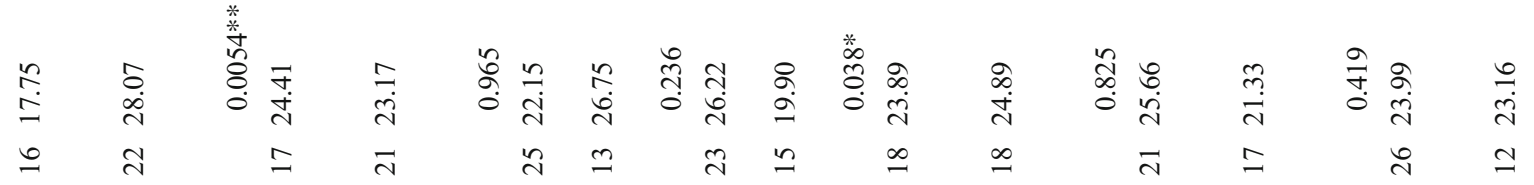

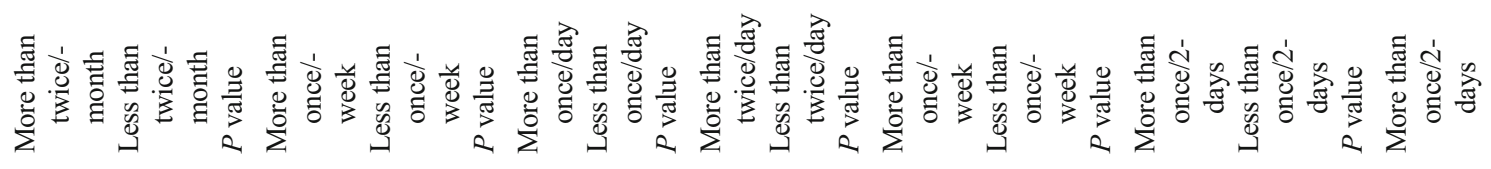

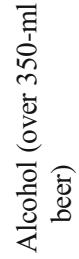
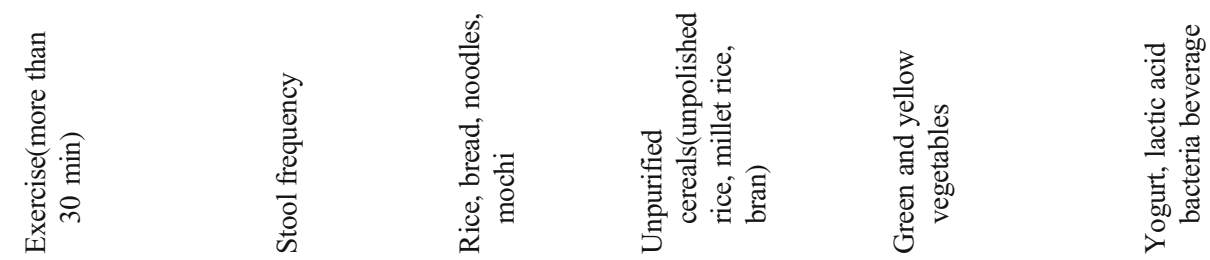
ACG GCG ACC ACC) and primer 2 (CAA GCA GAA GAC GGC ATA CGA). Library preparation was performed according to the $16 \mathrm{~S}$ library preparation Illumina protocol (Illumina). Libraries were sequenced using the MiSeq Reagent Kit v2 (500 Cycles) for 250-bp paired-ends (Fig. 2).

\section{Analysis of gut microbiota: taxonomy assignment based on the 16S rRNA gene sequence}

The paired-end reads of partial 16S rRNA gene sequences were clustered by $97 \%$ nucleotide identity, and then assigned taxonomic information using the Greengenes database (v13.8) (Second Genome, South San Francisco, CA) [20] through the Quantitative Insights into Microbial Ecology (QIIME) pipeline (v1.8.0) (Knight Lab, University of Colorado, Boulder, CO) [21]. The steps for data processing and assignment based on the QIIME pipeline were as follows: (i) joining paired-end reads, (ii) quality filtering with an accuracy of Q30 (>99.9\%) and a read length of $>300 \mathrm{bp}$, (iii) random extraction of 10,000 reads per sample for subsequent analysis, (iv) clustering of operational taxonomic units (OTUs) with 97\% identity by UCLUST (v1.2.22q) [22], and (v) assignment of taxonomic information to each OTU using RDP classifier [23] with the full-length 16S gene Greengenes data (v13.8) to determine the identity and composition of the bacterial genera. The analysis of the gut microbiota was contracted to Cykinso Inc., Tokyo, Japan.

From the results, the average of the 38 postmenopausal women was calculated at the phylum, class, order, family, and genus levels. Due to the exploratory nature of this study, only bacteria with an average proportion of $1 \%$ or more were subjected to statistical analysis, and bacteria with an average proportion of less than $1 \%$ were excluded (Fig. 1).

\section{Grouping by measurements of clinical parameters}

We divided age, menopausal age, BMD, fracture history, FRAX score, ucOC, TRACP-5b, vitamin $\mathrm{K} 1$, and vitamin $\mathrm{K} 2$ levels, into two groups as follows.

Age and BMD were divided based on the average values. This is because it was difficult to divide BMD based on the standard value for osteoporosis; only a few patients met the diagnostic criteria for osteoporosis after excluding those treated for osteoporosis during data collection. For menopausal age, the average value [24] for Japanese women (50.2 years) was used as the cutoff. Fracture history data was divided into groups with or without fracture history. For ucOC and TRACP-5b, the cutoffs of normal values in Japan were used. For vitamin K2, we used the lower limit of $0.06 \mathrm{ng} / \mathrm{mL}$ because the majority exceeded this value. In Japan, the recommended cutoff value of FRAX score is $15 \%$, but this study had only 3 out of 38 patients, and so it was difficult to divide them into 2 groups. The cutoff value was then taken as the average 


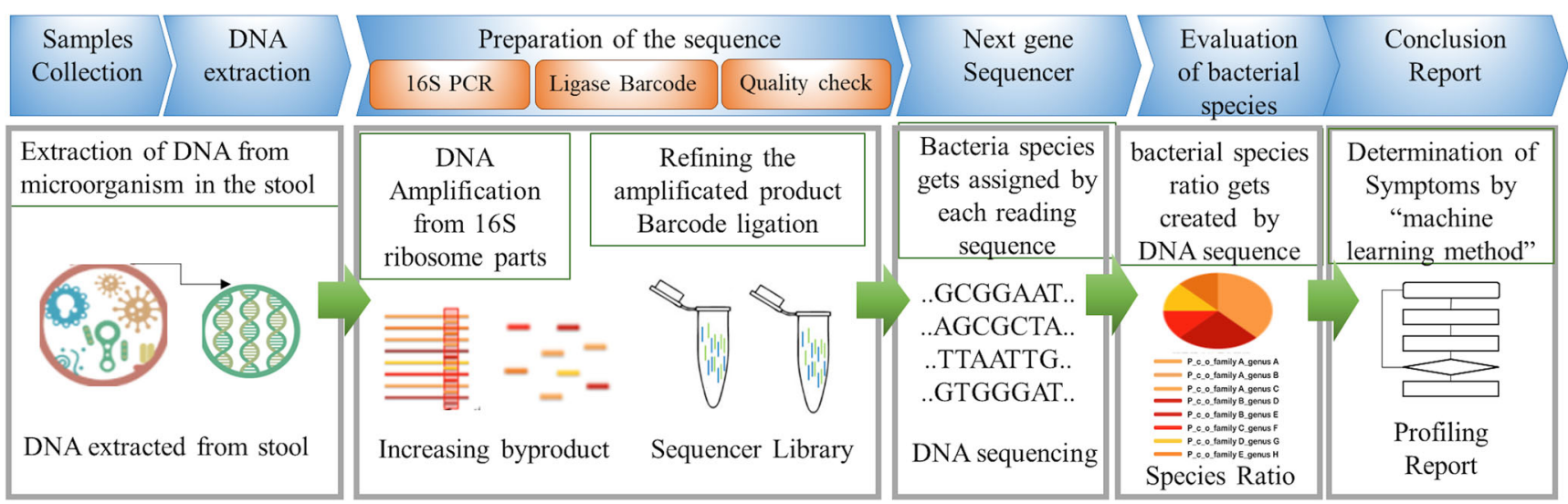

Fig. 2 Overview of fecal sample genetic profiling using the 16S rRNA gene to obtain a representation of gut microbiota composition

value. For natto, one intake was calculated as $40 \mathrm{~g}$ (vitamin $\mathrm{K}$ intake: $375.6 \mu \mathrm{g}[25]$ ), and the average intake of the subjects, $150 \mu \mathrm{g}$, was used as the cutoff value. From the results of the questionnaire, those who ate $40 \mathrm{~g}$ or more of natto 4 to 6 times a week were classified as the group with high frequency of natto intake, and those who ate natto 1 to 3 times a week were classified as the low frequency of natto intake group.

\section{Statistical analysis}

Comparisons were made to assess for significant differences in gut bacteria between groups. Statistical analysis was carried out using the Mann-Whitney $U$ test and $P<0.05$ was considered significant because more than half of the gut microbiota did not show a normal distribution. Fracture risk for each genus was determined from the two groups of gut microbiota and the fracture history, and odds ratios and relative risks were calculated. No correction for multiple testing was performed due to the exploratory nature of our study.

\section{Results}

The mean BMD was $87.05 \% \pm 11.78 \%$ for the YAM value and that for menopausal age was $51.79 \pm 4.65$ years. Bone metabolism markers (ucOC, TRACP-5b) and all assessed bone nutrients (vitamins $\mathrm{D}, \mathrm{K} 1, \mathrm{~K} 2, \mathrm{Ca}$, and $\mathrm{P}$ ) were within normal levels except for active vitamin D, which was slightly lower at $12.59 \pm 4.59 \mathrm{pg} / \mathrm{mL}$. FRAX score was $8.37 \% \pm 3.95 \%$ (Table 2). Among the 11 patients with a fracture history, 2 patients had fragility fracture (Fig. 3a). The questionnaire data showed that 17 of the 38 patients exercised at least once a week, 16 consumed alcohol more frequently, and 11 of them ate natto frequently (Table 1).

\section{Composition of gut microbial community}

The mean value for gut microbiota composition at the phylum level was $52.58 \% \pm 9.87 \%$ for Firmicutes, $32.83 \% \pm 11.64 \%$ for Bacteroidetes, $7.71 \% \pm 9.16 \%$ for Proteobacteria, and $5.66 \% \pm 4.51 \%$ for Actinobacteria (Fig. 3b).

\section{Age}

The patients were divided into groups aged $\geq 62.9$ and $<$ 62.9 years, $(n=20$ and $n=18$, respectively). The older group had significantly lower proportions of Lachnospiraceae and Blautia ( $P=0.047,0.002$, respectively) (Table 3).

\section{Menopausal age}

Age at menopause for Japanese women is 50.2 years, and so patients were divided into a high menopausal age $(n=22)$ and a low menopausal age group $(n=16)$. Oscillospira and Ruminococcus were significantly more abundant in the high

Table 2 Characteristics of the participants

\begin{tabular}{lc}
\hline Average value & \\
\hline Age & $62.87 \pm 6.22$ \\
Menopausal age & $51.79 \pm 4.65$ \\
BMD (YAM: \%) & $87.05 \pm 11.78$ \\
ucOC $(\mathrm{ng} / \mathrm{ml})$ & $4.38 \pm 2.16$ \\
TRACP $(\mathrm{mU} / \mathrm{dL})$ & $371.45 \pm 126.77$ \\
Vitamin D $(\mathrm{pg} / \mathrm{mL})$ & $12.59 \pm 4.59$ \\
Vitamin $\mathrm{K} 1(\mathrm{ng} / \mathrm{mL})$ & $1.08 \pm 0.75$ \\
VitaminK2 $(\mathrm{ng} / \mathrm{mL})$ & $0.08 \pm 0.08$ \\
FRAX $(\%)$ & $8.38 \pm 3.94$ \\
\hline
\end{tabular}

$B M D$, bone mineral density; $u c O C$, uncarboxylated osteocalcin; TRACP-5b, tartrate-resistant acid phosphatase $5 \mathrm{~b}$; $A L P$, alkaline phosphatase; $F R A X$, Fracture Risk Assessment 
Fig. 3 (a) Fracture history of the 38 patients in this study. b Mean values of gut microbiota composition at the phylum level
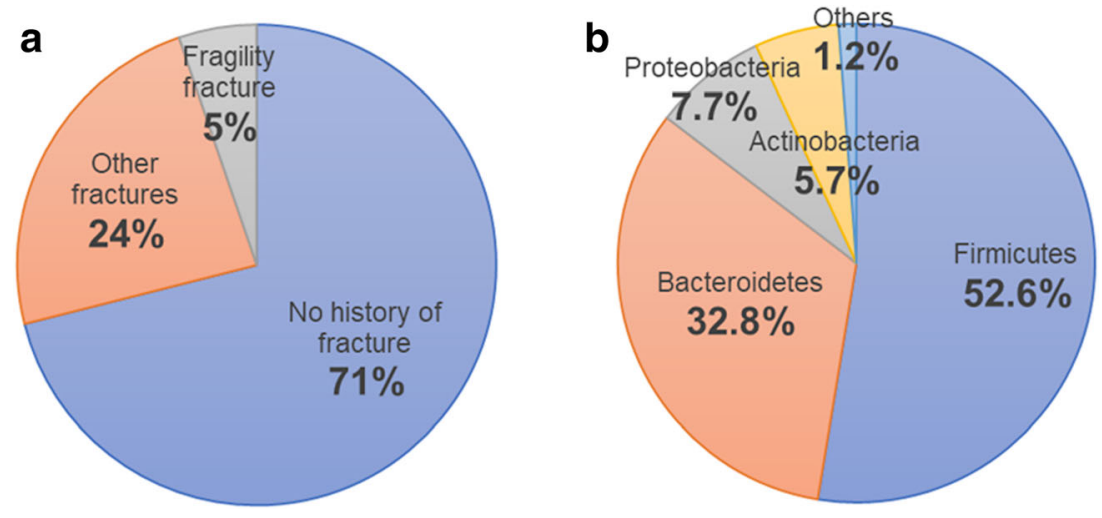

menopausal age group ( $P=0.018,0.014$, respectively). In contrast, Erysipelotrichaceae and Bifidobacterium were significantly abundant in the low menopausal age group $(P=$ $0.011,0.014$, respectively) (Table 3 ).

\section{BMD}

BMD was compared between the group with higher than the average BMD (high BMD group) and the group with lower than the average BMD (low BMD group; $n=19$ each). Rikenellaceae was significantly abundant in the low BMD group $(P=0.004)$ (Table 3$)$.

\section{Fracture history}

Fracture history was observed in 11 of the 38 patients; 2 of these had fragility fractures. Lachnospiraceae was less abundant in the group with fracture history $(P=0.02)$. Fracture history was not significantly affected by age (Table 3 ).

\section{ucOC}

The normal value for ucOC was set at $<4.5 \mathrm{ng} / \mathrm{mL}$, and thus $\geq 4.5 \mathrm{ng} / \mathrm{mL}$ was designated the high ucOC group and < $4.5 \mathrm{ng} / \mathrm{mL}$ was the low ucOC group ( $n=17$ and $n=11$, respectively). Sutterella was predominant in the high ucOC group $(P=0.044)$ (Table 3$)$.

\section{TRACP-5b}

Regarding TRACP-5b (normal value $<420 \mathrm{mU} / \mathrm{dL}$ ), patients were divided into a high TRACP-5b group and low TRACP$5 \mathrm{~b}$ group with a cutoff value of $420 \mathrm{mU} / \mathrm{dL}$ ( $n=11$ and $n=27$, respectively). Rikenellaceae and Bifidobacterium were predominant in the high TRACP-5b group $(P=0.013,0.016$, respectively) (Table 3 ).

\section{Vitamin K2}

The lower limit of blood vitamin K2 measurement was $0.06 \mathrm{ng} / \mathrm{mL}$, and so this was used as the cutoff value based on which patients were divided into high vitamin K2 groups $(n=10)$ and low vitamin K2 groups $(n=28)$. Bacteroides and Sutterella were predominant in the high vitamin K2 group $(P=0.022,0.026$, respectively) (Table 3$)$. In addition, there was no correlation between vitamin $\mathrm{K} 2$ and natto intake.

\section{FRAX}

The cutoff value for FRAX scores is $8.37 \%$; thus, patients were divided into a high and a low FRAX score groups ( $n=9$ and $n=29$, respectively). Veillonellaceae was predominant in the low FRAX score group $(P=0.044)$ (Table 3).

There was no significant difference between groups in age except for FRAX score. The high FRAX score group was predominantly older, because the FRAX tool also includes age as an item.

No significant difference was found in vitamin D, vitamin $\mathrm{K} 1$, ALP, Ca, and P.

\section{Fracture risk}

The incidence of fracture in the high fracture risk and low fracture risk groups was compared among the above bacteria. In the genus Bacteroides, the low-fracture risk group had a fracture incidence of $52.9 \%$, while the highfracture risk group had a fracture incidence of $9.5 \%$ (odds ratio $10.7,95 \%$ confidence interval: $1.88,60.9, P=$ 0.0049 , relative risk 5.57 . But the other bacteria showed no significant difference. Lachnospiraceae tended to show a lower risk of fracture, similar to the FRAX results above, and Rikenellaceae and Erysipelotrichaceae tended to show a slightly higher risk of fracture (Fig. 4). 


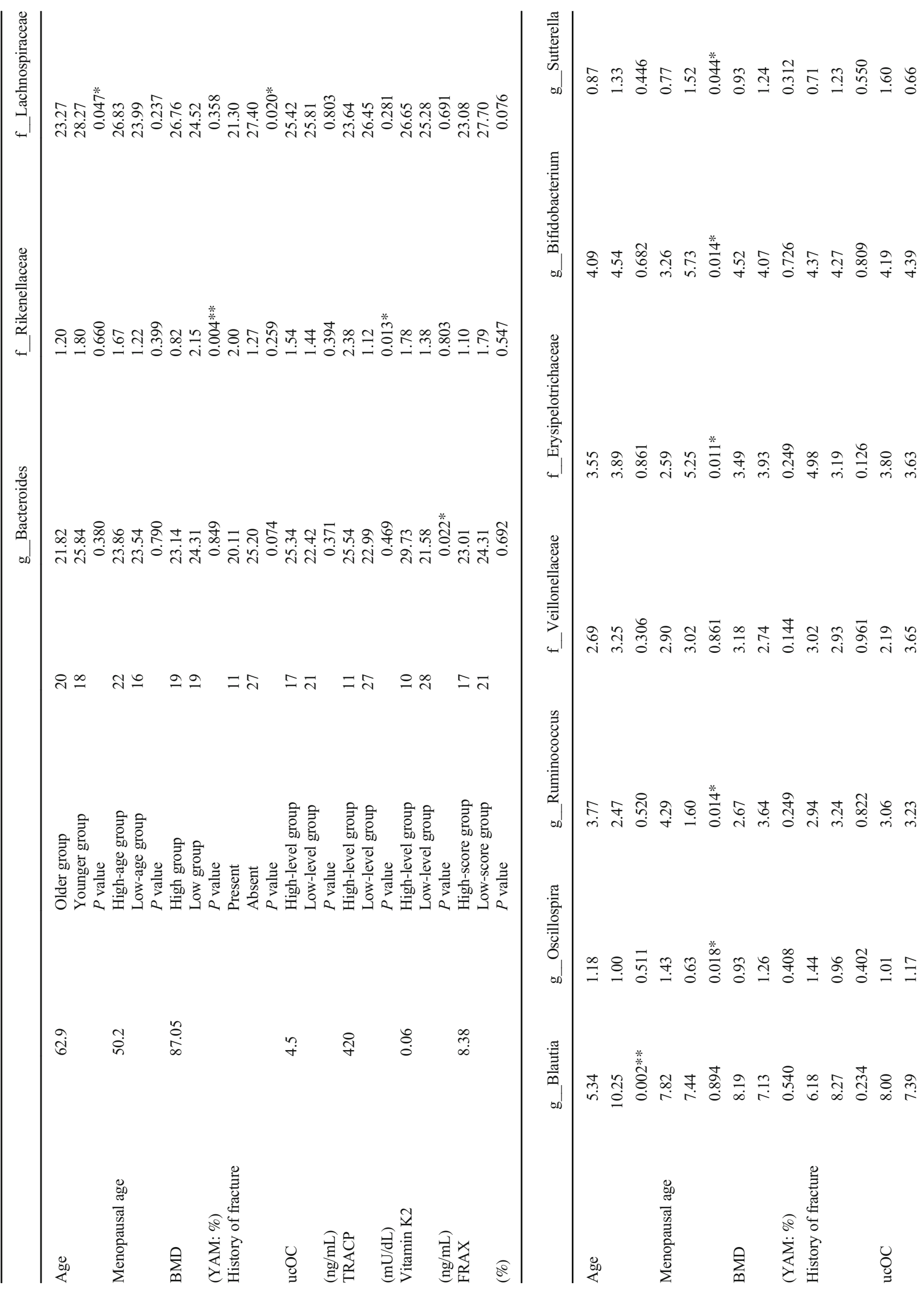




\section{Discussion}

In this study, the results of the statistical analysis suggest that gut bacteria affect bone mineral density and risk of fracture. Bacteroides showed a significant difference in vitamin $\mathrm{K} 2$ levels and fracture risk, and Rikenellaceae showed a significant difference in BMD and TRACP-5b levels. In addition, we would like to consider Lachnospiraceae, which is of importance in terms of both age and fracture history.

\section{Bacteroides}

Bacteroides synthesize vitamin K. Essentially, vitamin $\mathrm{K}$ is a group of several structurally similar, fat-soluble compounds required by humans for complete synthesis of certain proteins including phylloquinone (vitamin K1) and menaquinone ( $\mathrm{MKn}$; vitamin $\mathrm{K} 2)$. Vitamin $\mathrm{K}$ is a cofactor in the production of blood coagulation factors in the liver, osteocalcin in bone, and matrix Gla protein in cartilage and the vascular wall [26]. Furthermore, certain proteins in bone are vitamin $\mathrm{K}$-dependent, such as osteocalcin and matrix Gla protein, and thus vitamin $\mathrm{K}$ also plays an important role in regulating bone matrix quality [27].

Menaquinone-4 (MK-4, menatetrenone), a vitamin K2 homolog, is used in the treatment of osteoporosis in Japan. It is known to have well-recognized preventive effects in terms of bone resorption and fracture. In addition, the incidence of femoral, cervical, and vertebral compression fracture is higher among elderly women with low blood vitamin $\mathrm{K}$ concentrations and in those with high serum ucOC levels [28].

MKn contains a large amount of MK-7 found in Japanese natto, the well-known fermented soybean product containing Bacillus subtilis. MK-7 is also found in other fermented foods. MKn is also produced by the gut microbiota, while MK-10 and MK-11 are synthesized by Bacteroides [29].

In our study, Bacteroides was significantly more abundant in the high-level vitamin $\mathrm{K} 2$ group than in the low-level vitamin $\mathrm{K} 2$ group $(P=0.022)$ (Table 3$)$, but there was no significant difference in terms of natto intake between these two groups. Therefore, we surmised that dietary intake did not affect vitamin K2 level and that vitamin K2-producing Bacteroides exerted some influence.

Given that there was no significant difference in BMD, we postulated that Bacteroides does not affect bone strength. In addition, Bacteroides is believed to have some effect on bone quality and is associated with fracture unrelated to osteoporosis, rather than fragility fracture.

In our study, the fracture risk was 5.6 times higher in the low-level Bacteroides group (Fig. 4b), suggesting a role for Bacteroides in bone metabolism and fracture risk. It has also been reported that intake of meals that contain resistant starch, high levels of soluble fiber, and red meat can increase 


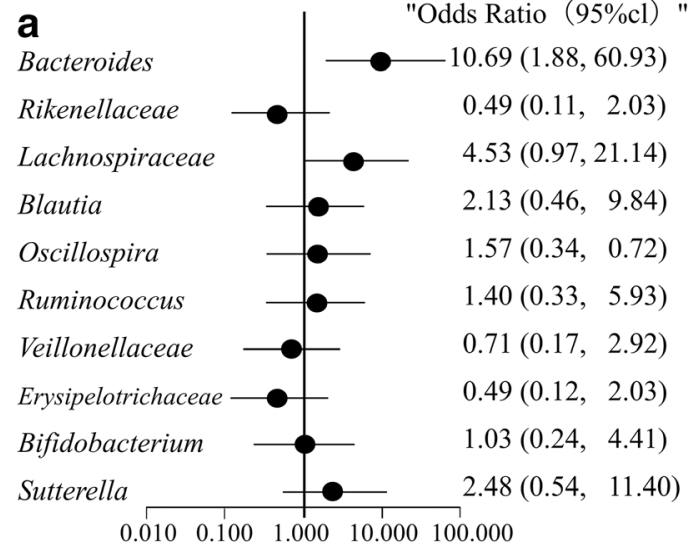

b

Bacteroides

Rikenellaceae

Lachnospiraceae

Blautia

Oscillospira

Ruminococcus

Veillonellaceae

Erysipelotrichaceae

Bifidbacterium

Sutterella

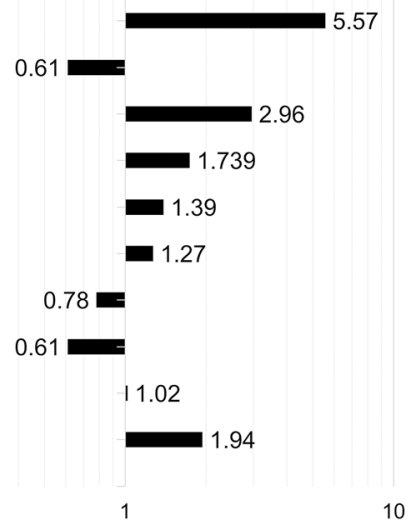

10

Fig. 4 Comparison of fracture incidence between the high- and low-isolation rate groups of gut microbiota. a Odds ratio (b) Relative risk: indicating fracture risk when these bacteria isolation rates are low. The higher the number [[isolation rate?]], the higher the fracture risk in the low-level group

the abundance of Bacteroides [7]. Therefore, ingestion of the above diets may reduce fracture risk.

\section{Rikenellaceae}

The functions of Rikenellaceae are not yet well understood. However, this family has been reported to be abundant in many diabetic patients [30]. Also, PD-1-/- mice, which are less likely to develop ulcerative colitis, showed changes in the composition of the intestinal microbiota and significantly reduced proportions of Rikenellaceae [31]. In our study, Rikenellaceae was more abundant in the low BMD group and in the high TRACP- 5 b group, suggesting that this family may have a negative effect on bone resorption and bone density. Furthermore, mice fed a high-fat diet were reported to show increased proportion of Rikenellaceae [32], so refraining from a high-fat diet may have a positive effect on bone strength even though this is an animal study.

\section{Lachnospiraceae}

Lachnospiraceae is among the most abundant bacteria [33] in the human gut. Lachnospiraceae breaks down complex polysaccharides into short-chain fatty acids. The abundance of these bacteria boosts host immunity and is a strong indicator of gut health. They are considered to be among the useful bacteria, because they are decreased in the intestine of the elderly [34] and in individuals with various diseases, such as diabetes mellitus [35], liver cirrhosis [36], and colon cancer [37].

It has also been reported that Lachnospiraceae was less abundant in individuals with low BMD and was positively correlated with BMD and T-score [38]. In this study, there was no significant difference in BMD, but the fracture incidence was significantly higher while the relative risk was three times higher, in the low Lachnospiraceae group than in the high Lachnospiraceae group (Fig. 4b). This suggested that
Lachnospiraceae may act as useful bacteria for predicting fracture risk.

\section{Conclusion}

Our data suggested that the abundance of Bacteroides and Lachnospiraceae may play a positive role in bone metabolism and fracture risk. Moreover, Rikenellaceae may have a negative effect on bone metabolism and fracture risk.

Attention to new treatments targeting particular species in gut microbiota is warranted for osteoporosis and fracture prevention. Further research should focus on analyzing specific species and their potential roles in increasing bone strength or preventing fractures.

\section{Limitations}

This study has some limitations that must be acknowledged. First, the study was conducted at a single-center in Japan, and so the subjects were Japanese nationals only. Thus, our findings may not be generalizable to other nationalities. Second, no dietary restrictions or lifestyle control measures were applied, which might have introduced some level of bias and affected our results. Third, the sample size was small, and so our study may not have sufficient statistical power for a definite conclusion. Fourth, the microbial taxa data in this study had a high variance, and thus our methods for statistical analysis may not have been ideal. Therefore, further multi-center studies with a larger sample size, and taking into account these and other limitations are warranted.

Acknowledgments We thank Yu Sawai, Satoshi Watanabe, and Aya Takeda of Cykinso Co. as well as Kayoko Shimonishi of Musashi Pharmacy for their services. Moreover, we express our gratitude to Florence Orim Ene for English proofreading and discussion, and 
Kumiko Okada and Maki Takahashi for taking blood specimens and collecting data. Daiya Ozaki, $\mathrm{MD}, \mathrm{PhD}$, takes responsibility for the integrity of the data analysis.

Funding This project received funding from the Musashiurawa Orthopedic and Internal Medicine Clinic.

\section{Compliance with ethical standards}

Conflicts of interest The authors declare that they have no conflicts of interest.

Ethical approval The study was performed in accordance with ethical standards as laid down in the World Medical Association's Declaration of Helsinki 1964 and its later amendments.

Informed consent Each participant gave written informed consent prior to inclusion in this study.

Open Access This article is licensed under a Creative Commons Attribution-NonCommercial 4.0 International License, which permits any non-commercial use, sharing, adaptation, distribution and reproduction in any medium or format, as long as you give appropriate credit to the original author(s) and the source, provide a link to the Creative Commons licence, and indicate if changes were made. The images or other third party material in this article are included in the article's Creative Commons licence, unless indicated otherwise in a credit line to the material. If material is not included in the article's Creative Commons licence and your intended use is not permitted by statutory regulation or exceeds the permitted use, you will need to obtain permission directly from the copyright holder. To view a copy of this licence, visit http:// creativecommons.org/licenses/by-nc/4.0/.

\section{References}

1. Yoshimura N, Muraki S, Oka H, Mabuchi A, En-Yo Y, Yoshida M, Saika A, Yoshida H, Suzuki T, Yamamoto S, Ishibashi H, Kawaguchi H, Nakamura K, Akune T (2009) Prevalence of knee osteoarthritis, lumbar spondylosis and osteoporosis in Japanese men and women: the research on osteoarthritis/osteoporosis against disability study. J Bone Miner Metab 27:620-628. https://doi.org/ 10.1007/s00774-009-0080-8

2. Orimo H, Nakamura T, Hosoi T et al (2015) [Japanese 2015 guidelines for prevention and treatment of osteoporosis.]:4-5[Article in Japanese] http://www.josteo.com/ja/guideline/doc/15_1.pdf

3. Kanis JA (2007) Assessment of osteoporosis at the primary health care level. Technical Report World Health Organization Collaborating Centre for Metabolic Bone Diseases; University of Sheffield, United Kingdom. https://www.sheffield.ac.uk/FRAX/

4. Iki M (2012) Epidemiology of osteoporosis in Japan. Clin Calcium 22:797-803 [Article in Japanese]

5. Frank DN, Amand ALS, Feldman RA, Boedeker EC, Harpaz N, Pace NR (2007) Molecular-phylogenetic characterization of microbial community imbalances in human inflammatory bowel diseases. Proc Natl Acad Sci U S A 21(104):13780-13785. https:// doi.org/10.1073/pnas.0706625104

6. Tap J, Mondot S, Levenez F, Pelletier E, Caron C, Furet JP, Ugarte E, Muñoz-Tamayo R, Paslier DLE, Nalin R, Dore J, Leclerc M (2009) Towards the human intestinal microbiota phylogenetic core. Environ Microbiol 11:2574-2584. https://doi.org/10.1111/j.14622920.2009.01982.x
7. Bibbò S, Ianiro G, Giorgio V, Scaldaferri F, Masucci L, Gasbarrini A, Cammarota G (2016) The role of diet on gut microbiota composition. Eur Rev Med Pharmacol Sci 20:4742-4749 https://www. europeanreview.org/article/11780

8. Swidsinski A, Loening-Baucke V, Lochs H, Hale LP (2005) Spatial organization of bacterial flora in normal and inflamed intestine: a fluorescence in situ hybridization study in mice. World $\mathrm{J}$ Gastroenterol 11:1131-1140. https://doi.org/10.3748/wjg.v11.i8. 1131

9. Rao VP, Poutahidis T, Ge Z, Nambiar PR, Horwitz BH, Fox JG, Erdman SE (2006) Proinflammatory CD4+ CD45RB(hi) lymphocytes promote mammary and intestinal carcinogenesis in Apc(min/+) mice. Cancer Res 66:57-61. https://doi.org/10.1158/ 0008-5472.CAN-05-3445

10. Kimura I, Ozawa K, Inoue D, Imamura T, Kimura K, Maeda T, Terasawa K, Kashihara D, Hirano K, Tani T, Takahashi T, Miyauchi S, Shioi G, Inoue H, Tsujimoto G (2013) The gut microbiota suppresses insulin-mediated fat accumulation via the shortchain fatty acid receptor GPR43. Nat Commun 4:1829. https://doi. org/10.1038/ncomms 2852

11. Wen L, Ley RE, Volchkov PY, Stranges PB, Avanesyan L, Stonebraker AC, Hu C, Wong FS, Szot GL, Bluestone JA, Gordon JI, Chervonsky AV (2008) Innate immunity and intestinal microbiota in the development of type 1 diabetes. Nature 455: 1109-1113. https://doi.org/10.1038/nature07336

12. Marin IA, Goertz JE, Ren T, Rich SS, Onengut-Gumuscu S, Farber E, Wu M, Overall CC, Kipnis J, Gaultier A (2017) Microbiota alteration is associated with the development of stress-induced despair behavior. Sci Rep 7:43859. https://doi.org/10.1038/srep43859

13. Sampson TR, Debelius JW, Thron T et al (2016) Gut microbiota regulate motor deficits and neuroinflammation in a model of Parkinson's disease. Cell 167:1469-1480.e12. https://doi.org/10. 1016/j.cell.2016.11.018

14. Kim YG, Udayanga KG, Totsuka N, Weinberg JB, Núñez G, Shibuya A (2014) Gut dysbiosis promotes M2 macrophage polarization and allergic airway inflammation via fungi-induced PGE2. Cell Host Microbe 15:95-102. https://doi.org/10.1016/j.chom. 2013.12.010

15. Wang J, Wang Y, Gao W, Wang B, Zhao H, Zeng Y, Ji Y, Hao D (2017) Diversity analysis of gut microbiota in osteoporosis and osteopenia patients. Peer J 5:e3450. https://doi.org/10.7717/peerj. 3450

16. Geirnaert A, Wang J, Tinck $M$ et al (2015) Interindividual differences in response to treatment with butyrate-producing Butyricicoccus pullicaecorum 25-3T studied in an in vitro gut model. FEMS Microbiol Ecol 91:fiv054. https://doi.org/10.1093/ femsec/fiv054

17. Roux AL, El Sayed F, Duffiet $P$ et al (2015) Ruminococcus gnavus total hip arthroplasty infection in a 62-year-old man with ulcerative colitis. J Clin Microbiol 53:1428-1430. https://doi.org/10.1128/ JCM.03040-14

18. Ando A (2015) Gut microbiota as a new organ. Clin J Gastroenterol 112:1939-1946(B). [Article in Japanese]. https://doi.org/10.11405/ nisshoshi.112.1939

19. Halleen JM, Alatalo SL, Janckila AJ et al (2001) Serum tartrateresistant acid phosphatase $5 \mathrm{~b}$ is a specific and sensitive marker of bone resorption. Clin Chem 47:597-600. https://doi.org/10.1093/ clinchem/47.3.597

20. DeSantis TZ, Hugenholtz P, Larsen N et al (2006) Greengenes, a chimera-checked 16S rRNA gene database and workbench compatible with ARB. Appl Environ Microbiol 72:5069-5072. https:// doi.org/10.1128/AEM.03006-05

21. Caporaso JG, Kuczynski J, Stombaugh J, Bittinger K, Bushman FD, Costello EK, Fierer N, Peña AG, Goodrich JK, Gordon JI, Huttley GA, Kelley ST, Knights D, Koenig JE, Ley RE, Lozupone CA, McDonald D, Muegge BD, Pirrung M, Reeder J, 
Sevinsky JR, Turnbaugh PJ, Walters WA, Widmann J, Yatsunenko T, Zaneveld J, Knight R (2010) QIIME allows analysis of highthroughput community sequencing data. Nat Methods 7:335-336. https://doi.org/10.1038/nmeth.f.303

22. Edgar RC (2010) Search and clustering orders of magnitude faster than BLAST. Bioinformatics. 26:2460-2461. https://doi.org/10. 1093/bioinformatics/btq461

23. Cole JR, Wang Q, Cardenas E, Fish J, Chai B, Farris RJ, KulamSyed-Mohideen AS, McGarrell DM, Marsh T, Garrity GM, Tiedje JM (2009) The Ribosomal Database Project: improved alignments and new tools for rRNA analysis. Nucleic Acids Res 37:D141D145. https://doi.org/10.1093/nar/gkn879

24. Kono S, Sunagawa Y, Higa H, Sunagawa H (1990) Age of menopause in Japanese women: trends and recent changes. Maturitas 12: 43-49. https://doi.org/10.1016/0378-5122(90)90059-F

25. Kamao M, Suhara Y, Tsugawa N et al (2007) Vitamin K content of foods and dietary vitamin $\mathrm{K}$ intake in Japanese young women. J Nutr Sci Vitaminol 53:464-470. https://doi.org/10.3177/jnsv.53. 464

26. Schurgers LJ, Teunissen KJ, Hamulyák K, Knapen MH, Vik H, Vermeer C (2007) Vitamin K-containing dietary supplements: comparison of synthetic vitamin $\mathrm{K} 1$ and natto-derived menaquinone-7. Blood 109:3279-3283. https://doi.org/10.1182/ blood-2006-08-040709

27. Guss JD, Taylor E, Rouse Z et al (2019) The microbial metagenome and bone tissue composition in mice with microbiome-induced reductions in bone strength. Bone 127:146-154. https://doi.org/10. 1093/ajcn/69.1.74

28. Feskanich D, Weber P, Willett WC, Rockett H, Booth SL, Colditz GA (1999) Vitamin K intake and hip fractures in women: a prospective study. Am J Clin Nutr 69:74-79. https://doi.org/10.1093/ ajcn/69.1.74

29. Fusaro M, Mereu MC, Aghi A, Iervasi G, Gallieni M (2017) Vitamin K and bone. Clin Cases Miner Bone Metab 14:200-206. https://doi.org/10.11138/ccmbm/2017.14.1.200

30. Zhang H-h, Liu J, Lv Y-j, Jiang Y-l, Pan J-x, Zhu Y-j, Huang M-g, Zhang S-k (2019) Changes in intestinal microbiota of type 2 diabetes in mice in response to dietary supplementation with instant tea or matcha. Can J Diabetes 44:44-52. https://doi.org/10.1016/j.jcjd. 2019.04.021
31. Park SJ, Kim J-H, Kim J-H et al (2017) PD-1 deficiency protects experimental colitis via alteration of gut microbiota. BMB Rep 50(11):578-583. https://doi.org/10.5483/BMBRep.2017.50.11.165

32. Hannelore D, Amin MG, David B at al. (2014) High-fat diet alters gut microbiota physiology in mice. ISME J 8:295-308. https://doi. org/10.1038/ismej.2013.155

33. Kurakawa T, Ogata K, Matsuda K, Tsuji H, Kubota H, Takada T, Kado Y, Asahara T, Takahashi T, Nomoto K (2015) Diversity of intestinal Clostridium coccoides group in the Japanese population, as demonstrated by reverse transcription-quantitative PCR. PLoS One 10:e0126226. https://doi.org/10.1371/journal.pone.0152753

34. Rondanelli M, Giacosa A, Faliva MA, Perna S, Allieri F, Castellazzi AM (2015) Review on microbiota and effectiveness of probiotics use in older. World J Clin Cases 3:156-162. https:// doi.org/10.12998/wjcc.v3.i2.156

35. Larsen N, Vogensen FK, van den Berg FW et al (2010) Gut microbiota in human adults with type 2 diabetes differs from non-diabetic adults. PLoS One 5:e9085. https://doi.org/10.1371/journal.pone. 0009085

36. Kakiyama G, Pandak WM, Gillevet PM, Hylemon PB, Heuman DM, Daita K, Takei H, Muto A, Nittono H, Ridlon JM, White MB, Noble NA, Monteith P, Fuchs M, Thacker LR, Sikaroodi M, Bajaj JS (2013) Modulation of the fecal bile acid profile by gut microbiota in cirrhosis. J Hepatol 58:949-955. https://doi.org/10.1016/j. jhep.2013.01.003

37. Ohigashi S, Sudo K, Kobayashi D, Takahashi O, Takahashi T, Asahara T, Nomoto K, Onodera H (2013) Changes of the intestinal microbiota, short chain fatty acids, and fecal $\mathrm{pH}$ in patients with colorectal cancer. Dig Dis Sci 58:1717-1726. https://doi.org/10. 1007/s10620-012-2526-4

38. Li C, Huang Q, Yang R, Dai Y, Zeng Y, Tao L, Li X, Zeng J, Wang Q (2019) Gut microbiota composition and bone mineral lossepidemiologic evidence from individuals in Wuhan, China. Osteoporos Int 30:1003-1013. https://doi.org/10.1007/s00198019-04855-5

Publisher's note Springer Nature remains neutral with regard to jurisdictional claims in published maps and institutional affiliations. 